\title{
Phenanthridine derivatives as potential HIV-1 protease inhibitors
}

\author{
PAVEL V. ERSHOV ${ }^{1,2}$, YURI V. MEZENTSEV ${ }^{1}$, LEONID A. KALUZHSKIY ${ }^{1}$ and ALEXIS S. IVANOV ${ }^{1}$ \\ ${ }^{1}$ Federal State Budgetary Institution, V.N. Orekhovich Research Institute of Biomedical Chemistry; \\ ${ }^{2}$ Federal State Budgetary Institution, Centre for Strategic Planning and Management of Biomedical Health Risks of \\ The Federal Medical Biological Agency, Moscow 119121, Russia
}

Received April 13,2020; Accepted September 14, 2020

DOI: $10.3892 / b r .2020 .1373$

\begin{abstract}
In the present study, the antiviral activity of phenanthridine derivatives was assessed. In total, the inhibitory effect of eight structurally similar low-molecular-weight hydrophobic compounds on HIV-1 protease (HIVp) was investigated. HIVp is a key enzyme in the HIV-1 life cycle. Surface plasmon resonance technology was used for affinity assessment of compounds binding with either monomeric or dimeric forms of HIVp. HIVp enzyme inhibition assays with chromogenic substrate VII were also used to determine the $\mathrm{IC}_{50}$ values. The most potent compound was 3,3,9,9-tetrameth yl-3,4,9,10-tetrahydro-2H,8H-phenanthridine-1,7-dione which binds to monomeric and dimeric forms of HIVp (apparent dissociation constant, 2-7 $\mu \mathrm{M} ; \mathrm{IC}_{50}, 36 \mu \mathrm{M}$ ), while possessing the most favorable Absorption, Distribution, Metabolism and Excretion parameters. Molecular docking simulations highlighted certain differences in the binding patterns of the phenanthridine derivatives with HIVp amino acid residues forming the flaps domain, monomer/monomer interfaces and the active site cavity of HIVp. Thus, it was hypothesized that the inhibitory effect of phenanthridine compounds on the enzymatic activity of HIVp may be due to restriction of substrate access to the HIVp active site.
\end{abstract}

\section{Introduction}

Characterization of novel biological activities of chemical compounds is closely associated with identification of potential drug prototypes. Phenanthridine derivatives are a group of low-molecular-weight (179-600 Da) non-peptide organic compounds, which are being actively studied, primarily due to their ability to bind efficiently with DNA and RNA (intercalators) (1) and also used as dyes (2). Representative compounds of this group have been shown to exhibit significant inhibitory

Correspondence to: Dr Pavel V. Ershov, Federal State Budgetary Institution, V.N. Orekhovich Research Institute of Biomedical Chemistry, 10 Building 8, Pogodinskaya, Moscow 119121, Russia E-mail: pavel79@inbox.ru

Key words: HIV1 protease, phenanthridine derivatives, surface plasmon resonance, monomer, dimer, enzyme inhibition activity against parasitic Leishmania protists (3), as well as activity against MCF-7, PC3 and HeLa tumor cell lines (4). Moreover, phenanthridine derivatives bind to specific target proteins both in vitro and in vivo, such as Bcl-XL (5), topoisomerase I (6) and poly (ADP-ribose) polymerases (7), causing anti-tumor and anti-apoptotic effects. Thus, phenanthridines present a class of compounds with a wide (but not fully studied) spectrum of biological and pharmacological properties.

The search for novel antiviral drug prototypes, including compounds against HIV-1, is one of the top priorities of pharmacological screening programs during the initial stages of drug discovery. In the present study, it was hypothesized that phenanthridine derivatives may exhibit biological activity against HIV-1, since one of the derivatives, 2,3,6,8-tetrachlorophenanthridine, has been characterized as a HIV-1 protease (HIVp) dimerization inhibitor, as shown in our previous studies $(8,9)$.

The aim of the present study was to assess the antiviral activity of eight new phenanthridine derivatives with homologous structures to 2,3,6,8-tetrachlorophenanthridine on HIVp. This viral enzyme is an obligatory dimer serving a key role in the HIV-1 life cycle, however a high rate of mutation during HIV-1 replication reduces the efficacy of chemotherapy (10-12).

Bioinformatics (structure-activity relationship predictions and molecular docking simulations), surface plasmon resonance (SPR) and enzyme inhibition assays were used to assess the activities of the derivatives. At least one of the new phenanthridine derivatives, 3,3,9,9-tetramethyl-3,4,9,1 0 -tetrahydro- $2 \mathrm{H}, 8 \mathrm{H}$-phenanthridine-1,7-dione (compound 2a), showed different affinities for the monomeric and dimeric forms of HIVp. Docking estimations with the lowest binding energy corresponded to the positioning of compound 2a in two regions of HIVp, the active site cavities and the flaps domain. Thus, it was suggested that binding of compound 2a restricted substrate access to the active site of HIVp due to the inhibitory effect of this compound.

\section{Materials and methods}

Recombinant proteins and chemical compounds. The purity of the recombinant HIVp (Bachem Holding AG) was $>95 \%$ as determined by SDS-PAGE. A micrOTOF-Q II (Bruker Corporation) mass-spectrometer was used as an additional 
quality control check of the protein preparation according to standard protocols of protein identification (data not shown) $(13,14)$. Chromogenic substrate VII, acetyl-pepstatin (a known competitive inhibitor of HIVp) (15) and the 'interfacial' hexapeptide Palmitoyl-Thr-Val-Ser-Tyr-Glu-Leu were obtained from Bachem Holding AG. A set of phenanthridine derivatives (Table I) was obtained from Asinex and Sigma-Aldrich; Merck KGaA. Protease inhibitors and phenanthridine derivatives were dissolved in $100 \%$ DMSO. Stock solutions were stored at $-20^{\circ} \mathrm{C}$. 1-ethyl-3-(3-dimethylaminopro pyl)-carbodiimide $\mathrm{HCl}, \mathrm{N}$-hydroxysuccinimide and $1 \mathrm{M}$ ethanolamine $\mathrm{HCl}$ ( $\mathrm{pH} 8.5$ ) were obtained from GE Healthcare. Maleic acid was obtained from Sigma-Aldrich; Merck KGaA.

Absorption, distribution, metabolism and excretion (ADME) and activity prediction for compounds. For prediction of ADME properties, the online resource SwissADME (swissadme.ch) (16) was used. Prediction of the activity spectrum for the phenanthridine derivatives against key HIV-1 enzymes (integrase, protease and reverse transcriptase) was performed using HIVprotI (bioinfo.imtech.res.in/manojk/hivproti/) (17).

SPR. Biacore 3000 and $8 \mathrm{~K}$ biosensors (GE Healthcare) were used for registration of intermolecular interactions. HIVp protein was covalently immobilized on the surface of a standard CM5 sensor chip (GE Healthcare) in sodium maleate buffer (pH 6.0) using a standard amine coupling protocol according to the manufacturer's protocol. The procedures for obtaining monomeric and dimeric forms of HIVp on the optical chip were performed as described previously $(8,9)$. Sodium acetate buffer (0.1 M, pH 5.0) containing $1 \mathrm{mM}$ EDTA and 3\% DMSO (v/v) was used as the running buffer. A biosensor channel without immobilized HIVp protein was used as a reference channel for subtraction of non-specific binding to the chip surface. The biosensor signal was recorded in resonance units (RU); one RU approximately corresponded to the binding of $1 \mathrm{pg}$ material per $1 \mathrm{~mm}^{2}$ chip surface area. Sensograms of association and dissociation of compound/HIVp complexes were processed separately with the calculation of association $\left(\mathrm{k}_{\mathrm{on}}\right)$ and dissociation rate $\left(\mathrm{k}_{\text {off }}\right)$ constant values using BIAevaluation version 4.1 (GE Healthcare). Apparent dissociation constant values $\left(\mathrm{K}_{\mathrm{d}}\right)$ were calculated as the $\mathrm{k}_{\text {off }} / \mathrm{k}_{\text {on }}$ ratio.

HIVp enzyme inhibition assay. To measure the enzymatic activity of HIVp, chromogenic substrate VII, with a maximum absorption spectra in the UV region (wavelength $300 \mathrm{~nm}$ ), was used. All measurements were performed at $25^{\circ} \mathrm{C}$ as described previously $(8,9)$.

Molecular docking simulations. DockPrep tools of UCSF Chimera version 1.14 (18-20) were used to prepare the PDB files of the crystallographic structure of HIVp 1Z1R (21) and phenanthridine derivatives for docking. Hydrogens as well as charges were added and water molecules were removed. Minimization procedure consisted of 300 steps with the steepest descent and conjugate gradient algorithms used (22). Molecular docking was performed using iGEMDOCK version 2.1 (23) with stable (slow) docking default settings (population size, 300; generations, 80; number of solutions, 10).

\section{Results}

Activity prediction for compounds and molecular docking simulations. The antiviral activity for the set of phenanthridine derivatives was determined for three main HIV-1 enzymes: Integrase, $\mathrm{HIV}-1$ protease and reverse transcriptase. $\mathrm{IC}_{50}$ values and percent inhibition values were predicted for all compounds using HIVprotI (Table I). The phenanthridine derivatives were predicted to exhibit activity against HIV-1p ( $\mathrm{IC}_{50}$ value range, 6-234 $\mu \mathrm{M}$ with $31-53 \%$ inhibition), as well as against the HIV-1 integrase and reverse transcriptase, although to a lesser extent.

Further molecular docking analysis of the phenanthridine derivatives over the entire surface of both monomeric and dimeric form of HIVp was performed using iGEMDOCK. Docking of HIVp dimerization inhibitor 2,3,6,8-tetrachlorophenanthridine $(8,9)$ was used as reference for analysis of binding to the HIVp monomer/monomer interface.

Together, the molecular simulations indicated that all phenanthridine derivatives tested docked in both the dimeric and monomeric forms of HIVp (Table II). Amino acid residues $\mathrm{Ile}^{50}$ of the dimeric, as well as $\mathrm{Val}^{32}, \mathrm{Ile}^{47}, \mathrm{Gly}^{48}, \mathrm{Gly}^{49}, \mathrm{Ile}^{84}$ and $\mathrm{Ile}^{54}$ of the monomeric form of HIVp were involved in van der Waals interactions with the phenanthridine derivatives (Table II). Subsequently, experimental validation of activities and binding models, predicted using bioinformatics tools, was performed using SPR and enzyme activity assays.

SPR. An optical biosensor test system, consisting of the immobilized monomeric or dimeric form of HIVp in the different flow cells $(8,9)$, was used to screen the binding of phenanthridine derivatives at a concentration of $100 \mu \mathrm{M}$. Only 3 compounds, compounds $2 \mathrm{a}, 4 \mathrm{a}$ and $5 \mathrm{a}$, showed positive binding signals with HIVp monomer and dimer exceeding 10 RU (cut-off level with correction for the baseline drift upon analyte injection). Furthermore, each of these compounds were injected at different concentrations to calculate the $\mathrm{K}_{\mathrm{d}}$ values of the compound/HIVp complexes (Table III). SPR binding sensograms of compound $2 \mathrm{a}$, which formed the most affine complexes with HIVp, are shown in Fig. S1.

Inhibition of HIVp activity by the phenanthridine derivatives. HIVp enzymatic activity was sensitive to the known inhibitors acetyl-pepstatin and the 'interfacial' hexapeptide. HIVp activity decreased by 60 and $35 \%$ compared to control (without any inhibitor) (data not shown), respectively, which indicated that biochemical testing provided relevant results. Among the eight phenanthridine derivatives tested, only compounds $2 \mathrm{a}$ and 4a demonstrated HIVp inhibitory activity (Table III). Fig. S2 shows HIVp activity data in the presence of different concentrations of compound $2 \mathrm{a}$. $\mathrm{IC}_{50}$ calculation for compound $2 \mathrm{a}$ is shown in Fig. 1.

\section{Discussion}

A flow chart of the experimental procedure used in the present study is shown in Fig. 2. Predicted using molecular docking, amino acid residues in positions 44-57 of the HIVp monomer represent the so-called flaps (or flaps domains) that are considered to be involved in regulation of the substrate access to the active site of the enzyme due to the different conformational states of 


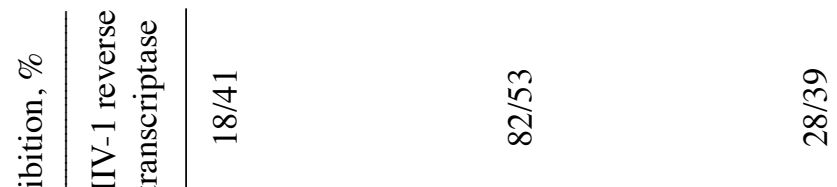

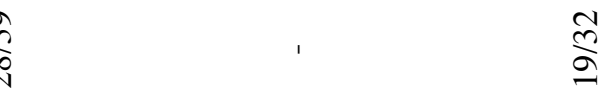
$\frac{2}{\infty} \sqrt{\infty}$
$\frac{\aleph}{2}$
$\stackrel{m}{=}$
$\frac{\mathbb{7}}{\frac{7}{\infty}}$
$\frac{n}{6}$
$\stackrel{\wp}{\Xi}$
$\underset{\infty}{\stackrel{Ð}{\ddagger}}$

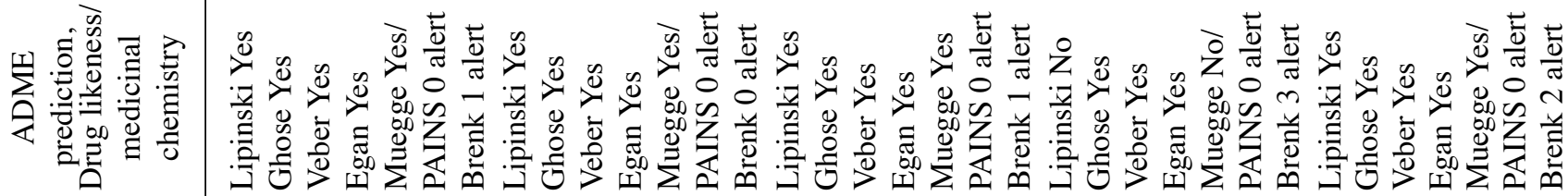
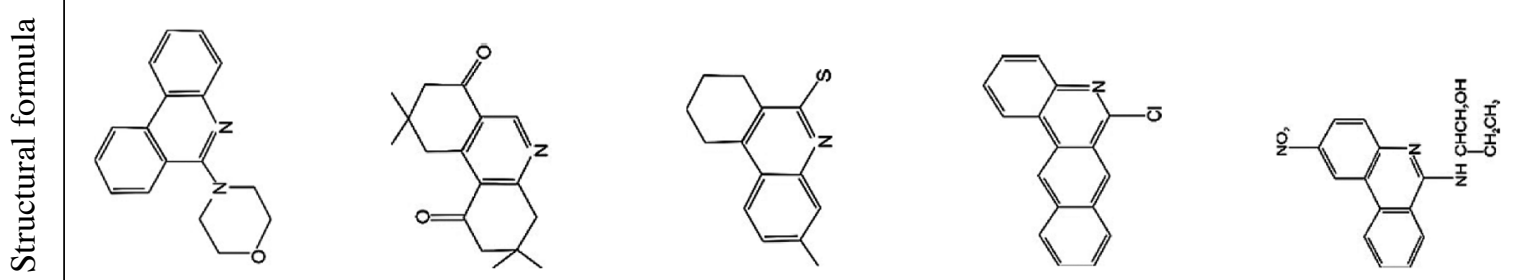

$\sqrt{2}$

สิ

ชิ

$\bar{m}$

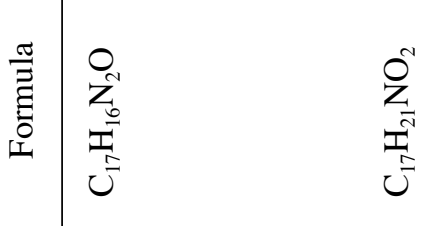

$u_{\substack{j \\ n}}^{n}$

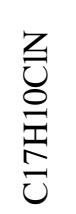

O̊
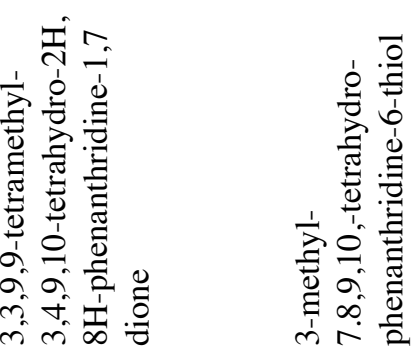

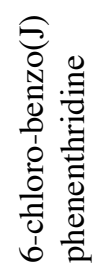

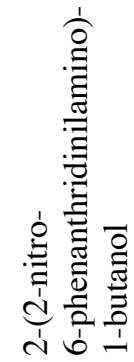

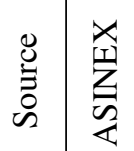

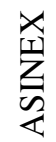

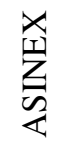

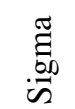

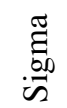

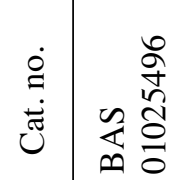

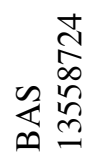

象空

๙ิ
तิ
ปิ

$\frac{n}{n}$

m

ซ

เ 


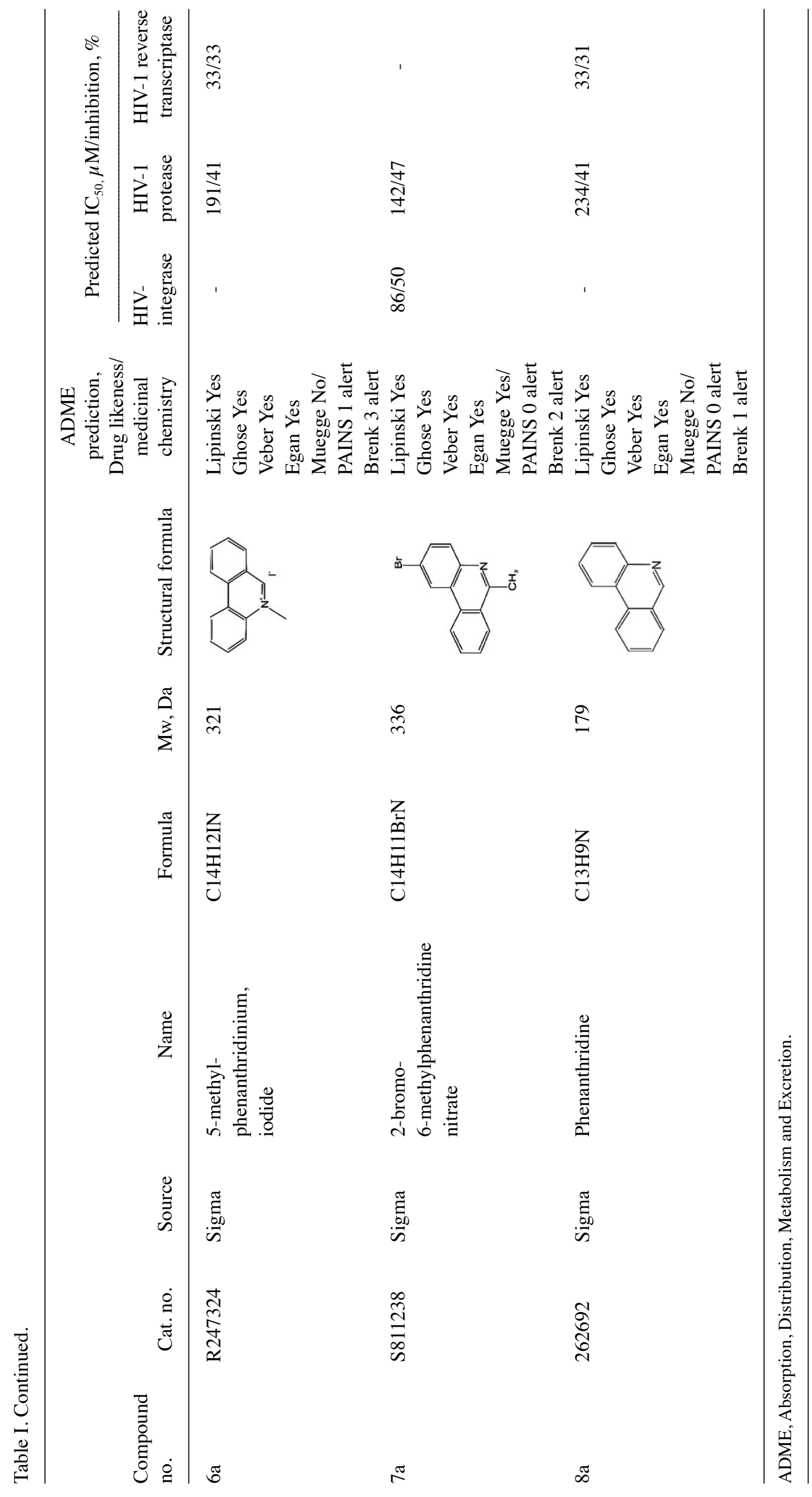




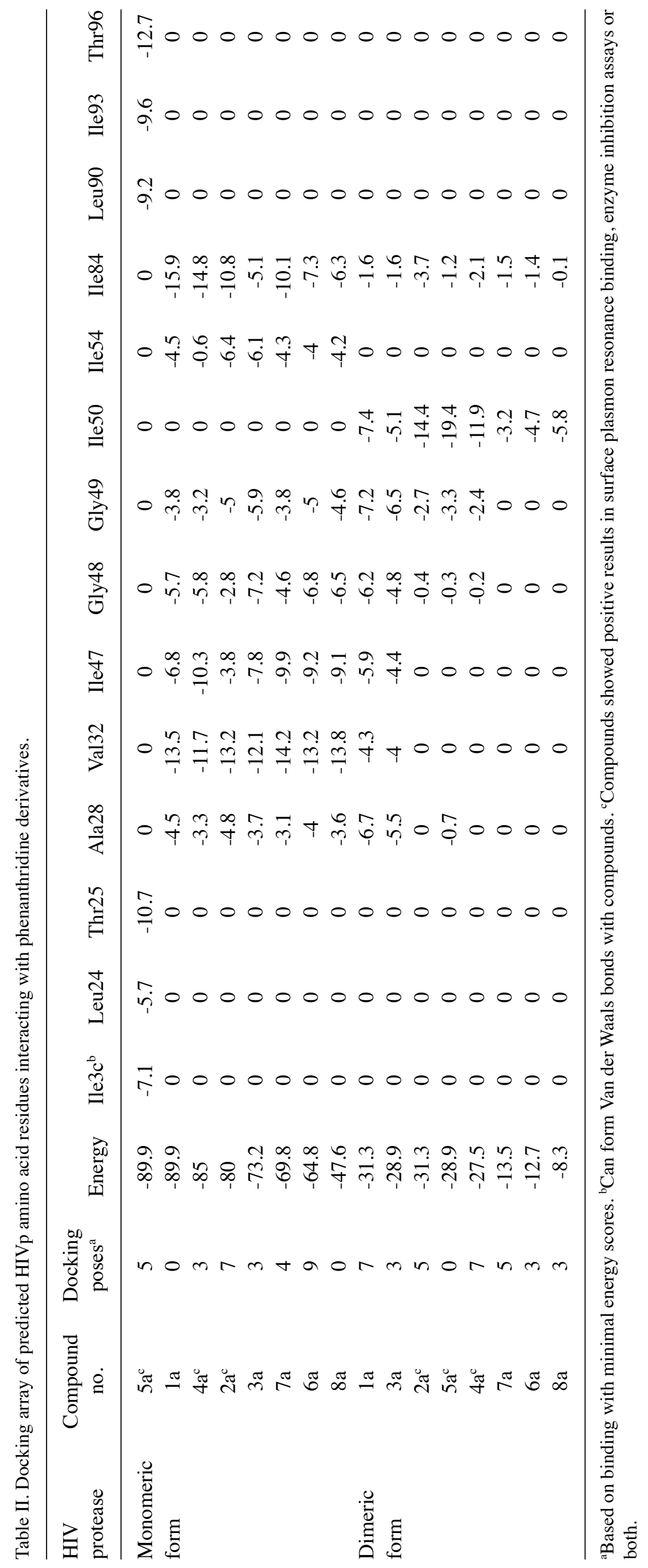


Table III. Summary of molecular docking simulations, surface plasmon resonance analysis and HIV protease enzyme inhibition assay for phenanthridine derivatives.

\begin{tabular}{|c|c|c|c|c|c|c|c|c|c|}
\hline Parameters & HIVp & $1 \mathrm{a}$ & $2 a$ & $3 a$ & $4 a$ & $5 \mathrm{a}$ & $6 a$ & $7 \mathrm{a}$ & $8 a$ \\
\hline \multirow[t]{2}{*}{ Surface plasmon resonance $\mathrm{K}_{\mathrm{d}}, \mu \mathrm{M}$} & Dimer & No & $\sim 7$ & No & $\sim 1,000$ & $\sim 500$ & No & No & No \\
\hline & Monomer & No & $\sim 2$ & No & $\sim 100$ & $\sim 300$ & No & No & No \\
\hline \multirow{3}{*}{$\begin{array}{l}\text { Enzyme innibition assay } \mathrm{IC}_{50}, \mu \mathrm{M} \\
\text { Binding regions of } \mathrm{HIVp}^{\mathrm{a}}\end{array}$} & & No & $\sim 36$ & No & $\sim 45$ & No & No & No & No \\
\hline & Dimer & II & II & II & II & I & II & II & II \\
\hline & Monomer & I, II & I, II & I, II & I, II & III & I, II & I, II & I, II \\
\hline
\end{tabular}

${ }^{a}$ According to the location of HIVp amino acid residues interacting with phenanthridine derivatives, predicted using docking analysis. I, active site cavity of HIVp; II, flaps domain; III, monomer/monomer interface region of HIVp; HIVp, HIV protease.

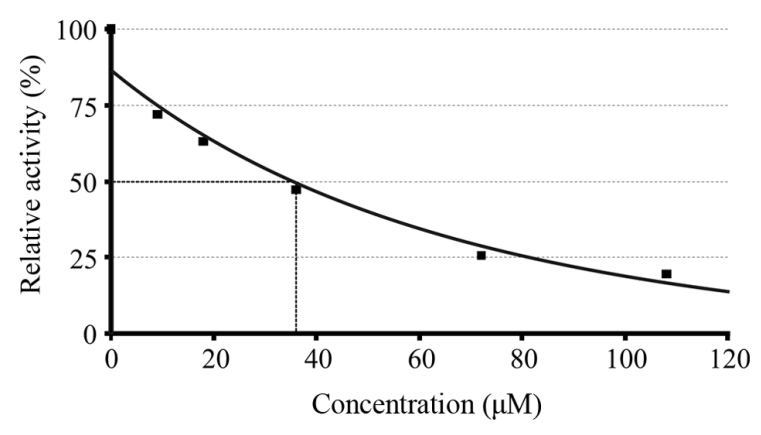

Figure 1. HIV-1 protease $\mathrm{IC}_{50}$ value calculation for compound $2 \mathrm{a}$.

the flaps (opened to closed) $(24,25)$. An exception to this was compound 5a, which, according to the docking model (Table SI), interacted with the monomeric form of HIVp within the monomer/monomer contact area ( $\mathrm{Pro}^{1} \mathrm{Gln}^{2} \mathrm{Ile}^{3} \mathrm{Thr}^{4}$ and $\mathrm{Thr}{ }^{961} \mathrm{Le}$ $\left.\mathrm{u}^{971} \mathrm{Asn}^{98} \mathrm{Phe}^{99 \prime}\right)(26,27)$. In addition, compound 5a was stabilized by the hydrophobic interactions with $\mathrm{Leu}^{241}, \mathrm{Thr}^{261}$ and $\mathrm{Leu}^{90}$, Ile $^{93}$ (Table II). A similar binding model for 2,3,6,8-tetrachlorophenanthridine was predicted (Table SI). An association between $\mathrm{K}_{\mathrm{d}}$ values and predicted binding models was observed. Interactions of compounds $2 \mathrm{a}, 4 \mathrm{a}$ and $5 \mathrm{a}$ with the monomeric form of HIVp exhibited notably lower values of predicted binding energy in the docking models compared with the dimeric form.

Compound $4 \mathrm{a}$ demonstrated a positive result in the HIVp enzyme inhibition assay ( $\mathrm{IC}_{50}$ value, $\left.45 \mu \mathrm{M}\right)$, had low binding affinity to the dimeric and monomeric forms of $\mathrm{HIVp}$ $\left(\mathrm{K}_{\mathrm{d}}, \sim 1 \times 10^{-4}\right.$ and $\sim 1 \times 10^{-5} \mathrm{M}$, respectively). Compound $2 \mathrm{a}$ showed $\mathrm{a} \mathrm{K}_{\mathrm{d}}$ and $\mathrm{IC}_{50}$ value of 2-7 and $36 \mu \mathrm{M}$, respectively. The binding affinity of compound $2 \mathrm{a}$ to the HIVp monomer $\left(\mathrm{K}_{\mathrm{d}} \sim 2 \mu \mathrm{M}\right)$ was $4 \mathrm{x}$ higher than 2,3,6,8-tetrachlorophenanthridine $\left(\mathrm{K}_{\mathrm{d}} \sim 9 \mu \mathrm{M}\right)(8,9)$. It is worth noting that the predicted binding sites for compound $2 \mathrm{a}$ were different from those for 2,3,6,8-tetrachlorophenanthridine which targeted the HIVp interface. In general, compound $2 \mathrm{a}$ had the most favorable ADME parameters among all the phenanthridine derivatives tested and thus may be used as the basic structure in the search for more potent drug prototypes aimed at inhibition of HIV-1p.

Additional SPR experiments showed no significant effect of the phenanthridine derivatives on the re-association of the HIVp dimeric form on the optical chip (Table SII). This indirectly indicated the absence of the compound's interference with the HIVp dimerization process. The optimal

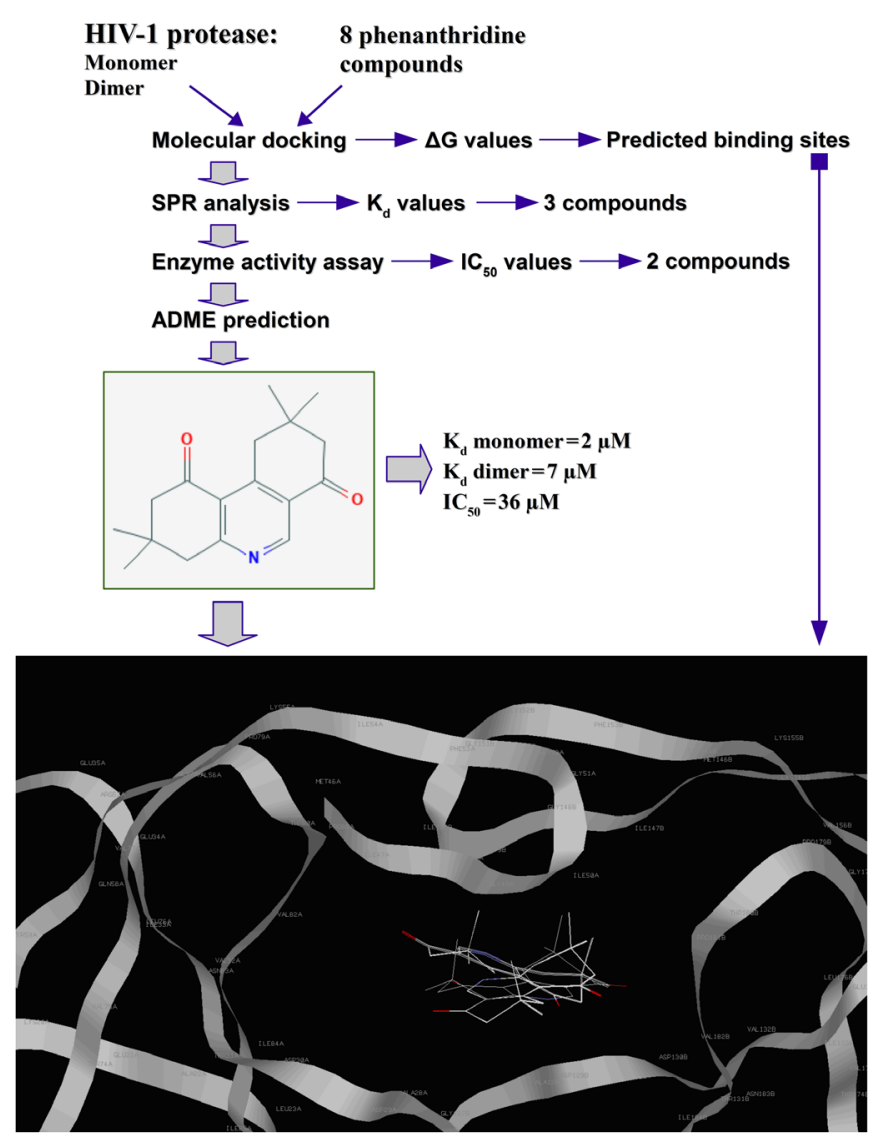

Figure 2. Flow chart of the experimental procedure used in the present study to identify the potential value of the phenanthridine derivatives as HIV-1 protease inhibitors, and the binding parameters and predicted binding site of compound 2a. SPR, surface plasmon resonance; $K_{d}$ apparent dissociation constant; ADME, Absorption, Distribution, Metabolism and Excretion.

docking conformations with the lowest binding energy values corresponded to the positioning of compound $2 \mathrm{a}$ in two regions of HIV-1p, the active site cavities and the flaps domain (Table III), and it was hypothesized that the inhibitory effect of compound $2 \mathrm{a}$ on HIV-1p protease activity may be due to the restriction of substrate access to the HIVp active site.

In conclusion, through the use of complex methodological approaches, including experimental and bioinformatics methods, it was demonstrated that the phenanthridine derivatives with different spatial arrangements of the substituent groups exhibited differing efficiencies of HIVp inhibition as 
well as different SPR binding profiles with the monomeric and dimeric forms of HIVp. Furthermore, according to the molecular docking simulations, phenanthridine/HIVp binding models varied significantly, indicating a difference in their potential mechanisms of action on HIVp. These results confirmed our hypothesis that phenanthridines, as a class of low-molecular-weight compounds, in addition to their already known beneficial biological and pharmacological activities against several different types of cancer, may additionally possess antiviral activity against HIV-1.

\section{Acknowledgements}

Not applicable.

\section{Funding}

This research was funded by the Program of Basic Scientific Research of National Science Academies for 2013-2020. Biosensor $8 \mathrm{~K}$ analysis and mass spectrometry quality control of protein preparation were funded by MINOBRNAUKI (agreement no. 075-15-2019-1502).

\section{Availability of data and materials}

The datasets used and/or analyzed during the present study are available from the corresponding author on reasonable request. Supplementary material are available at FigShare repository 10.6084/m9.figshare.12837563.

\section{Authors' contributions}

PVE conceived the study. PVE and YVM performed the experiments and wrote the manuscript. LAK and ASI analyzed and interpreted the data. All authors read and approved the final manuscript.

\section{Ethics approval and consent to participate}

Not applicable.

\section{Patient consent for publication}

Not applicable.

\section{Competing interests}

The authors declare that they have no competing interests.

\section{References}

1. Azad I, Ahmad R, Khan T, Saquib M, Hassan F, Akhter Y, Khan AR and Nasibullah M: Phenanthridine derivatives as promising new anticancer agents: Synthesis, biological evaluation and binding studies. Future Med Chem 12: 709-739, 2020.

2. Tumir LM, Radić Stojković M and Piantanida I: Come-back of phenanthridine and phenanthridinium derivatives in the 21st century. Beilstein J Org Chem 10: 2930-2954, 2014.

3. Fuchino H, Kawano M, Mori-Yasumoto K, Sekita S, Satake M, Ishikawa T, Kiuchi $\mathrm{F}$ and Kawahara N: In vitro leishmanicidal activity of benzophenanthridine alkaloids from Bocconia pearcei and related compounds. Chem Pharm Bull (Tokyo) 58: 1047-1050, 2010.
4. Wan M, Zhang L, Chen Y, Li Q, Fan W, Xue Q, Yan F and Song W: Synthesis and anticancer activity evaluation of novel phenanthridine derivatives. Front Oncol 9: 274, 2019.

5. Bernardo PH, Wan KF, Sivaraman T, Xu J, Moore FK, Hung AW, Mok HY, Yu VC and Chai CL: Structure-activity relationship studies of Phenanthridine-based Bcl-XL inhibitors. J Med Chem 51: 6699-6710, 2008.

6. Thai KM, Bui QH, Tran TD and Huynh TN: QSAR modeling on benzo[c]phenanthridine analogues as topoisomerase I inhibitors and anti-cancer agents. Molecules 17: 5690-5712, 2012.

7. Inbar-Rozensal D, Castiel A, Visochek L, Castel D, Dantzer F, Izraeli S and Cohen-Armon M: A selective eradication of human nonhereditary breast cancer cells by phenanthridine-derived polyADP-ribose polymerase inhibitors. Breast Cancer Res 11: R78, 2009.

8. Ershov PV, Gnedenko OV, Molnar AA, Lisitsa AV, Ivanov AS and Archakov AI: Biosensor analysis of the interaction of potential dimerization inhibitors with HIV-1 protease. Biomed Khim 55: 462-478, 2009 (In Russian).

9. Ershov PV, Gnedenko OV, Molnar AA, Lisitsa AV, Ivanov AS and Archakov AI: Kinetic and thermodynamic analysis of dimerization inhibitors binding to HIV protease monomers by surface plasmon resonance. Biomeditsinskaya Khimiya 58: 43-49, 2012.

10. Darke PL: Stability of dimeric retroviral proteases. Meth Enzymol 241: 104-127, 1994.

11. Weber IT, Kneller DW and Wong-Sam A: Highly resistant HIV-1 proteases and strategies for their inhibition. Future Med Chem 7: 1023-1038, 2015.

12. Weber IT and Agniswamy J: HIV-1 Protease: Structural perspectives on drug resistance. Viruses 1: 1110-1136, 2009.

13. Hao P, Ren Y and Xie Y: Label-free relative quantification method for Low-abundance glycoproteins in human serum by micrOTOF-Q. J Chromatogr B: Analyt Technol Biomed Life Sci 877: 1657-1666, 2009.

14. Purushothaman AK and Pemiah B: Ultra high performance liquid chromatography-ultraviolet-electrospray ionization-micrOTOF-Q II analysis of flavonoid fractions from Jatropha tanjorensis. Pharmacogn Mag 10 (Suppl 3): S472-S479, 2014.

15. Richards AD, Roberts R, Dunn BM, Graves MC and Kay J: Effective blocking of HIV-1 proteinase activity by characteristic inhibitors of aspartic proteinases. FEBS Lett 247: 113-117, 1989.

16. Daina A, Michielin O and Zoete V: SwissADME: A free web tool to evaluate pharmacokinetics, drug-likeness and medicinal chemistry friendliness of small molecules. Sci Rep 7: 42717, 2017.

17. Qureshi A, Rajput A, Kaur G and Kumar M: HIVprotI: An integrated web based platform for prediction and design of HIV proteins inhibitors. J Cheminform 10: 12, 2018.

18. Pettersen EF, Goddard TD, Huang CC, Couch GS, Greenblatt DM, Meng EC and Ferrin TE: UCSF Chimera-a visualization system for exploratory research and analysis. J Comput Chem 25: 1605-1612, 2004

19. Wang J, Wang W, Kollman PA and Case DA: Automatic atom type and bond type perception in molecular mechanical calculations. J Mol Graph Model 25: 247-260, 2006.

20. Shapovalov MV and Dunbrack RL: A smoothed Backbonedependent rotamer library for proteins derived from adaptive kernel density estimates and regressions. Structure 19: 844-858, 2011.

21. Martin JL, Begun J, Schindeler A, Wickramasinghe WA, Alewood D, Alewood PF, Bergman DA, Brinkworth RI, Abbenante G, March DR, et al: Molecular recognition of macrocyclic peptidomimetic inhibitors by HIV-1 protease. Biochemistry 38: 7978-7988, 1999.

22. Ortiz D, delToro D, Ordyan M, Pajak J, Sippy J, Catala A, Oh CS, Vu A, Arya G, Feiss M, et al: Evidence that a catalytic glutamate and an 'Arginine Toggle' act in concert to mediate ATP hydrolysis and mechanochemical coupling in a viral DNA packaging motor. Nucl Acid Res 47: 1404-1415, 2019.

23. Hsu KC, Chen YF, Lin SR and Yang JM: iGEMDOCK: A graphical environment of enhancing GEMDOCK using pharmacological interactions and Post-screening analysis. BMC Bioinformatics 12 (Suppl 1): S33, 2011.

24. Scott WR and Schiffer CA: Curling of flap tips in HIV-1 protease as a mechanism for substrate entry and tolerance of drug resistance. Structure 8: 1259-1265, 2000.

25. Yu Y, Wang J, Chen Z, Wang G, Shao Q, Shi J and Zhu W: Structural insights into HIV-1 protease flap opening processes and key intermediates. RSC Adv 7: 45121-45128, 2017.

26. Babé LM, Pichuantes S and Craik CS: Inhibition of HIV protease activity by heterodimer formation. Biochemistry 30: 106-111, 1991

27. Miller M, Schneider J, Sathyanarayana BK, Toth MV, Marshall GR, Clawson L, Selk L, Kent SB and Wlodawer A: Structure of complex of synthetic HIV-1 protease with a substrate-based inhibitor at 2.3 A resolution. Science 246: 1149-1152, 1989. 